\section{Military Technical College Kobry El-Kobbah, Cairo, Egypt}

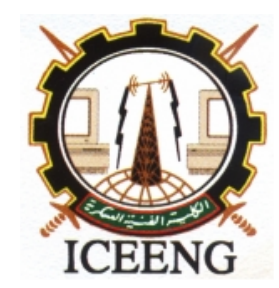

\section{$7^{\text {th }}$ International Conference on Electrical Engineering \\ ICEENG 2010}

\title{
Radar Matched Filter Based on Lattice Wave Digital Filter Approach
}

$$
\text { By }
$$

K. H. Moustafa*

M. A. Romeh**

H.Kamel***

\section{$\underline{\text { Abstract: }}$}

Based on the theory of wave digital filters (WDFs), a new model is derived to represent single pulse matched filters for radar applications. This matched filter was obtained by calculating the coefficients of Lattice Wave Digital Filter (LWDF) adaptors by getting filter transfer function that results from arbitrary amplitude transfer function. In this paper, we are interested only to the main loop of the matched filter frequency response and its location of pass-band edges.

* Assistant prof., Egyptian Armed Force, MTC, Cairo

** Egyptian Armed Force, MTC, Cairo

*** Ph.D, Egyptian Armed Force, MTC, Cairo 


\section{Introduction:}

In most cases, a band-pass filter characteristic is obtained based on lowpass-to-bandpass frequency transformation starting from a normalized and known lowpass transfer function. This frequency transformation controls the location of passband edges and, moreover, transfers zero frequencies, completely. This may impose design compromises, if the transformation would result in a sub optimal characteristic. The design procedures using the frequency transformation techniques having several disadvantages: - Computational complexity is required to transform the lowpass (highpass) specifications into band-pass ones and inversely.

- Non-optimality results from the impossibility to approximate different losses in the two stop-bands which in the case in different applications.

- Degree restriction for lattice structures results from the impossibility to design a bandpass lattice filter with degree $n$ such that $n / 2$ becomes an even number.

There is an increasing need for high-speed real time digital filters for applications such as video processing, and radar signal processing. WDFs have well known properties, which make them attractive for this task [3-5]. These filters exhibit good dynamic range performance and low sensitivity that make them suitable for use with very short coefficient word lengths. Wave digital Filters have been proved to be very robust with a guaranteed linear stability even for poles very close to the unit circle.

In this paper, the design of bandpass lattice wave digital matched filter is described. This filter is obtained by calculating the coefficients of Lattice Wave Digital Matched Filter (LWDMF) adaptors by getting filter transfer function that results from arbitrary amplitude transfer function. By this way, it is possible to design a band-pass filter transfer function which cannot be obtained based on low-pass filter frequency transformation.

The conventional matched filter requires $\sin (\mathrm{x}) / \mathrm{x}$ frequency response, where $\mathrm{x}=\left(\omega-\omega_{0}\right) \delta / 2, \omega_{0}$ is the central frequency and $\delta$ is the pulse width [7]. The proposed realization of LWDMF is simulated using the MATLAB simulator. The MATLAB program is used as tool to get the transfer function of Chebychev bandpass LWDF from arbitrary loss specifications (i.e. passband frequency $F_{p}$, stopband frequency $F_{s}$, maximum passband ripples $A_{p}$, minimum stopband attenuations $A_{s}$, and sampling frequency). 


\section{The Resulting Transfer Function H(s):}

In this paper, we have chosen a Multiple-target tracking instrumentation radar (also called MOTR) [7] with the following parameters; frequency $\mathrm{f}_{0}=5.4 \mathrm{GHz}$, and pulse width $\delta=0.685 \mathrm{nsec}$. Assume the sampling frequency $\mathrm{f}_{\mathrm{s}}=16 \mathrm{GHz}$ according to the sampling theory. From the above data, the bandwidth is given by $\Delta \mathrm{f}=(1.37 / \delta)=2$ $\mathrm{GHz}$ [1]. We also obtain the following parameters: $\mathrm{f}_{1}=4.49 \mathrm{GHz}, \mathrm{f}_{2}=6.49 \mathrm{GHz}$, $\mathrm{A}_{\mathrm{P}}=0.5 \mathrm{~dB}$, and $\mathrm{A}_{\mathrm{s}}=50 \mathrm{~dB}$ where $f_{1}, f_{2}, A_{P}$, and $A_{s}$ are two cut-off frequencies of the bandpass filter, maximum pass-band ripples, and minimum stop-band attenuations respectively. We have chosen a (nearly) symmetrical Chebychev bandpass filter. Based on [2], the order of this filter is determined to be 6 .

From the bandpass filter specifications mentioned above, we can get the filter transfer function either in $S$ or $Z$ domains. Then, we get the transfer function, $\mathrm{H}(\mathrm{s})$, of Chebychev bandpass filter. Also, we obtain the roots of its denominator as shown in Table 1.

$H(s)=0.0112 /\left[\left(S^{2}+0.0928 S+0.6641\right)\left(S^{2}+0.1566 S+0.4556\right)\left(S^{2}+0.0638 S+0.3126\right)\right]$

\begin{tabular}{|l|l|}
\hline$S_{1}=-0.0464+0.8136 i$ & $S_{2}=-0.0464-0.8136 i$ \\
\hline$S_{3}=-0.0783+0.6704 i$ & $S_{4}=-0.0783-0.6704 i$ \\
\hline$S_{5}=-0.0319+0.5582 i$ & $S_{6}=-0.0319-0.5582 i$ \\
\hline
\end{tabular}

Table 1. Roots of the Chebychev bandpass transfer function

Characteristic frequency values resulting from the approximation are shown in Figure 1. For the desired case, we interested only to the main loop of the matched filter frequency response and its location of pass-band edges.

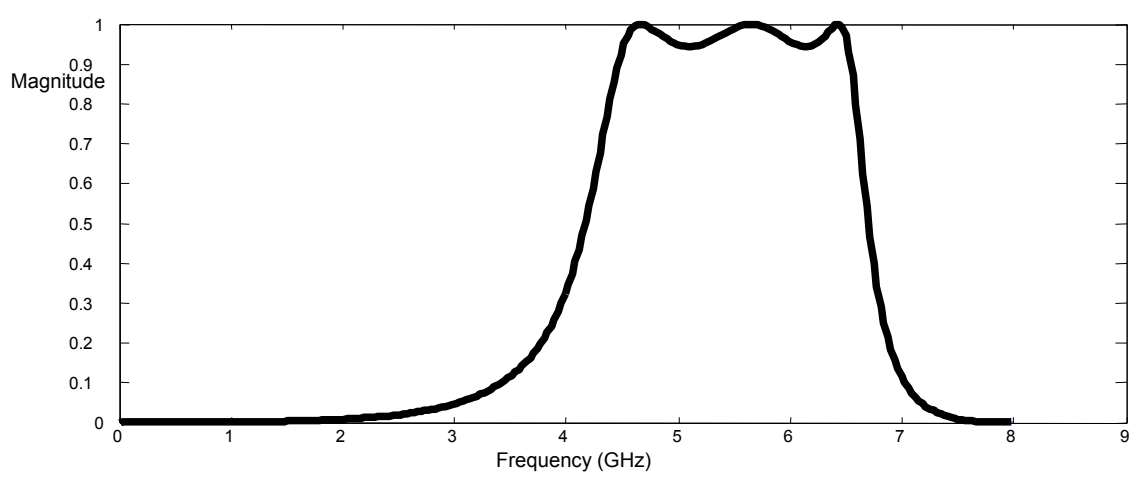

Figure 1. The Chebychev approximation resulting from the specifications 
Then, we compare this response with $\sin (\mathrm{x}) / \mathrm{x}$ response as shown in Figure 2.

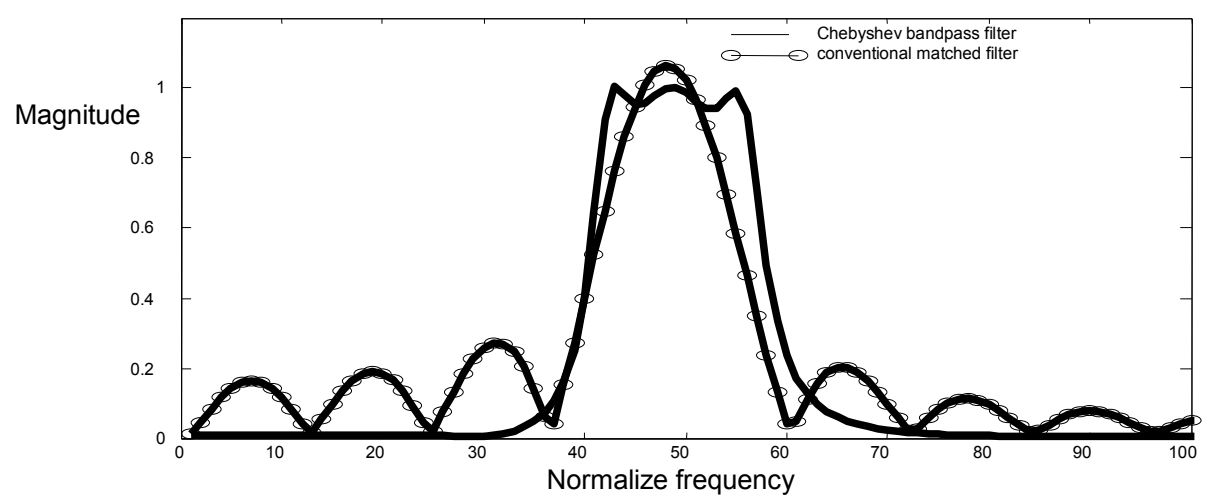

Figure 2. Comparing the Chebychev response with approximation $\sin (\mathbf{x}) / \mathbf{x}$ response

It is clear that there is a big difference between both of them. This is followed by an optimization step as shown in Figure 3.

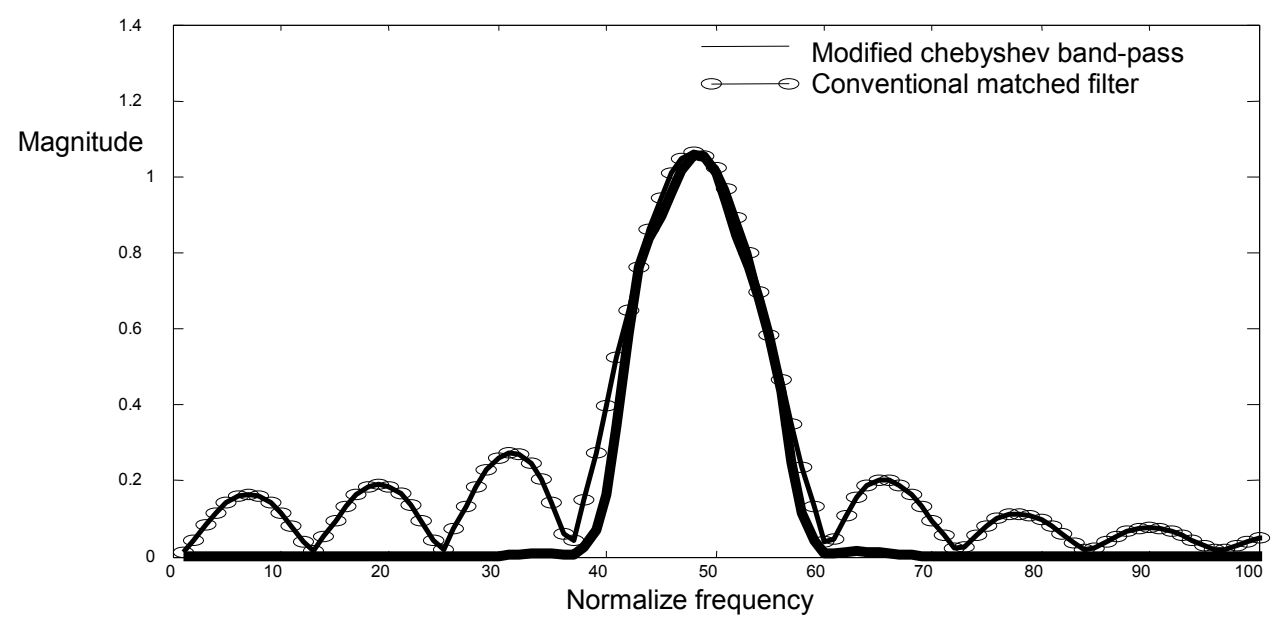

Figure 3. Optimization of the Chebychev response with approximation $\sin (\mathrm{x}) / \mathrm{x}$ response

We get the correspondence transfer function, $\mathrm{H}(\mathrm{z})$, of a modified Chebychev band-pass filter as follows:

$\begin{aligned} H(z)= & {\left[0.027742-0.083226 Z^{-2}+0.083226 Z^{-4}-0.027742 Z^{-6}\right] /\left[1+2.8083 Z^{-1}+4.4426 Z^{-2}+4.5088 Z^{-3}+3.2318 Z^{-4}+\right.} \\ & \left.1.453 Z^{-5}+0.37833 Z^{-6}\right] .\end{aligned}$

Based on $[4,6]$, we build the correspondence $6^{\text {th }}$ order LWDMF. The LWDMF is a cascade of all-pass sections of second order. This filter has three sections. The all-pass sections consist of delay elements and, hence, are called adaptors. Two-port adaptors that have two inputs and two outputs are considered. Different structure types for the adaptors are used, depending on the range of gamma $\gamma$ coefficients to a second coefficient, designated as the alpha $a$ coefficient. The alpha coefficient is always 
positive and in the range 0 to 0.5 . In our desired filter, the number of both coefficients and adaptors is 6 . The gamma $\gamma$ coefficients, adaptor type, and alpha $\alpha$ coefficients of the single pulse LWDMF are shown in Table 2.

\begin{tabular}{|l|l|l|l|l|}
\hline Stage & Name & Adaptor Type & Alpha & $\begin{array}{l}\text { Multiplication } \\
\text { coefficient }\end{array}$ \\
\hline 1 & Adaptor (1) & Type(4) & $\alpha_{1}=0.1057$ & $\gamma_{1}=-0.89427$ \\
\hline 2 & Adaptor (2) & Type(2) & $\alpha_{2}=0.20181$ & $\gamma_{2}=0.20181$ \\
\hline 3 & Adaptor (3) & Type(4) & $\alpha_{3}=0.1943$ & $\gamma_{3}=-0.80573$ \\
\hline 4 & Adaptor (4) & Type(2) & $\alpha_{4}=0.37399$ & $\gamma_{4}=0.37399$ \\
\hline 5 & Adaptor (5) & Type(4) & $\alpha_{5}=0.0926$ & $\gamma_{5}=-0.9074$ \\
\hline 6 & Adaptor (6) & Type(1) & $\alpha_{6}=0.4763$ & $\gamma_{6}=0.52374$ \\
\hline
\end{tabular}

Table 2. Single pulse LWDMF parameters

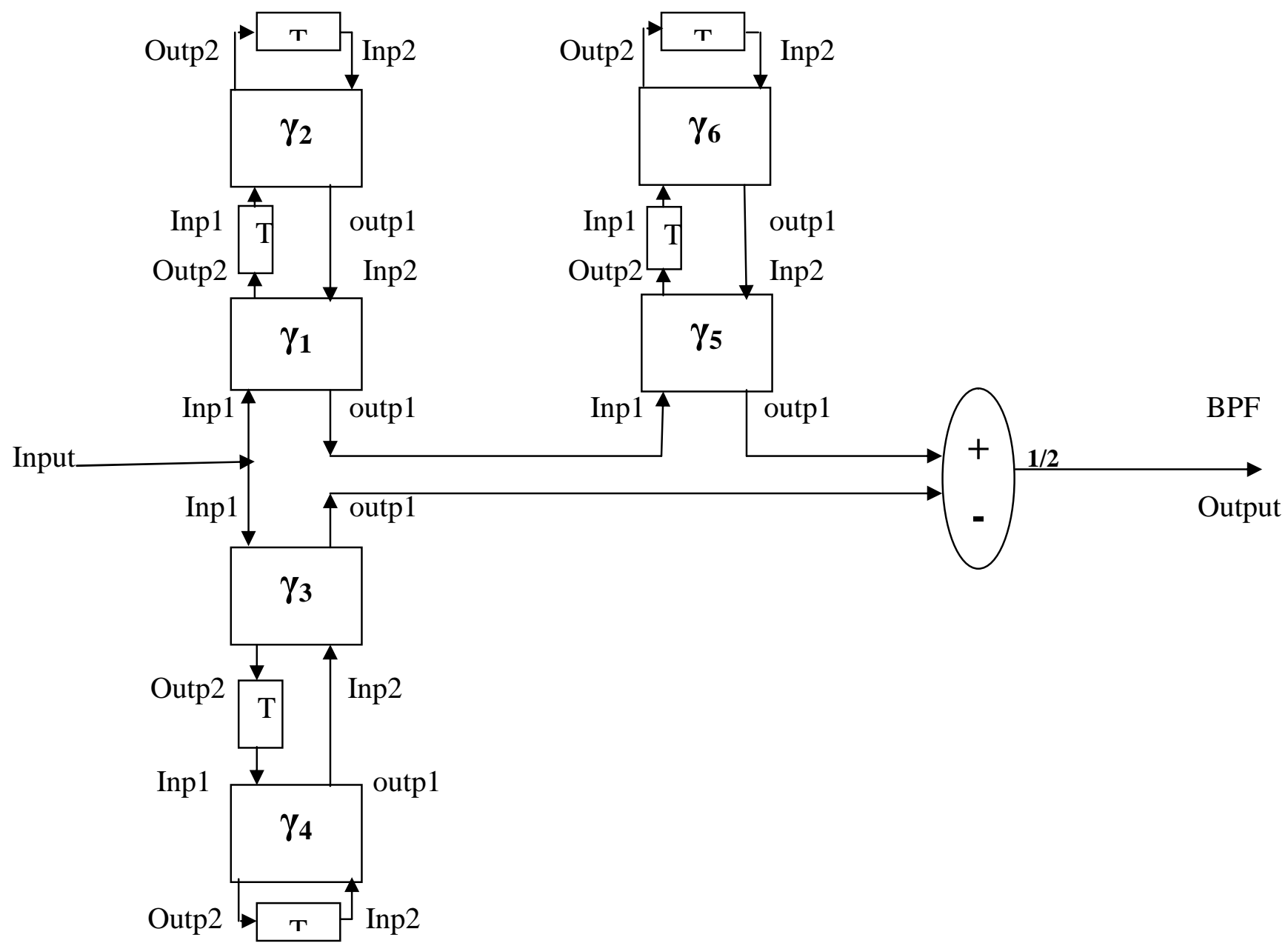

Figure 4. Signal flow diagram of $6^{\text {th }}$ order chebyshev band-pass LWDMF 


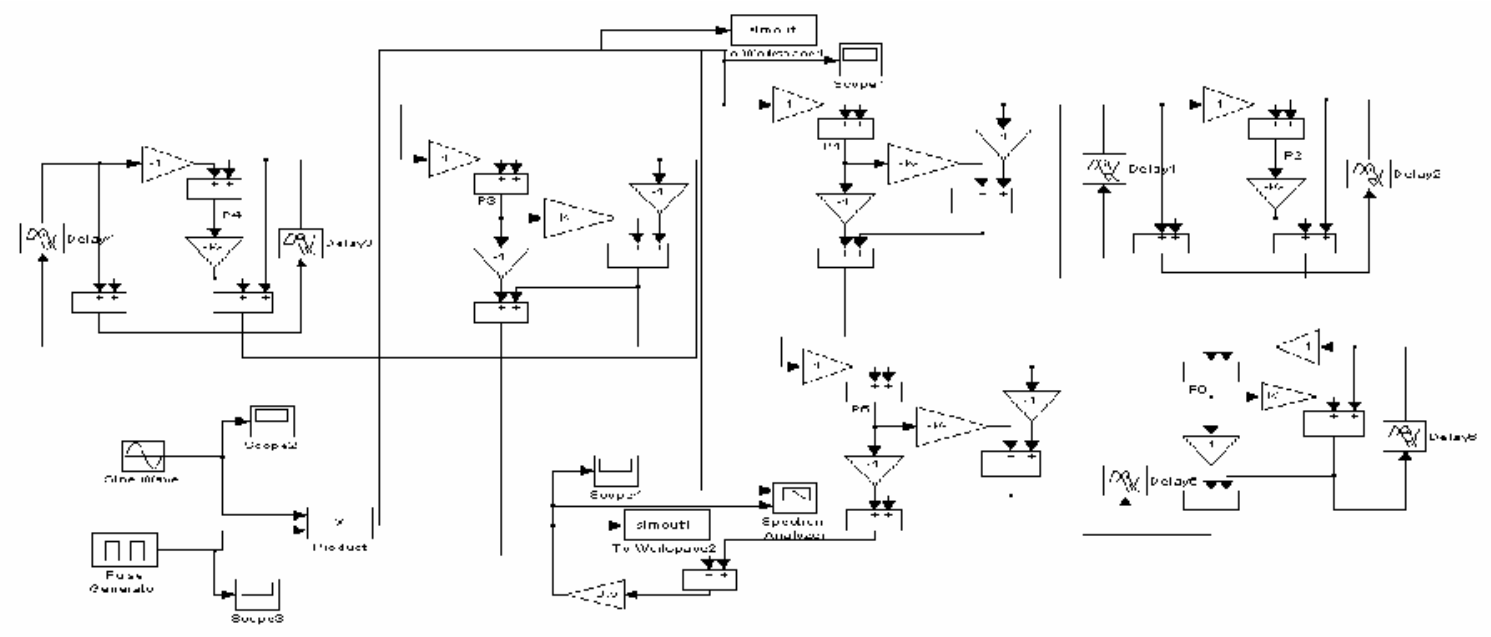

Figure 5. Interconnections for $6^{\text {th }}$-order chebychev band-pass LWDMF

Based on [6], the signal flow diagram for this WDF is shown in Figure 4, and the interconnections with the adaptor structure are shown in Figure 5.

We now check the matching of this filter by applying an input as a pulsed RF signal with a frequency $\mathrm{f}_{0}=5.4 \mathrm{GHz}$ and a pulse width $\delta=0.685 \mathrm{nsec}$.

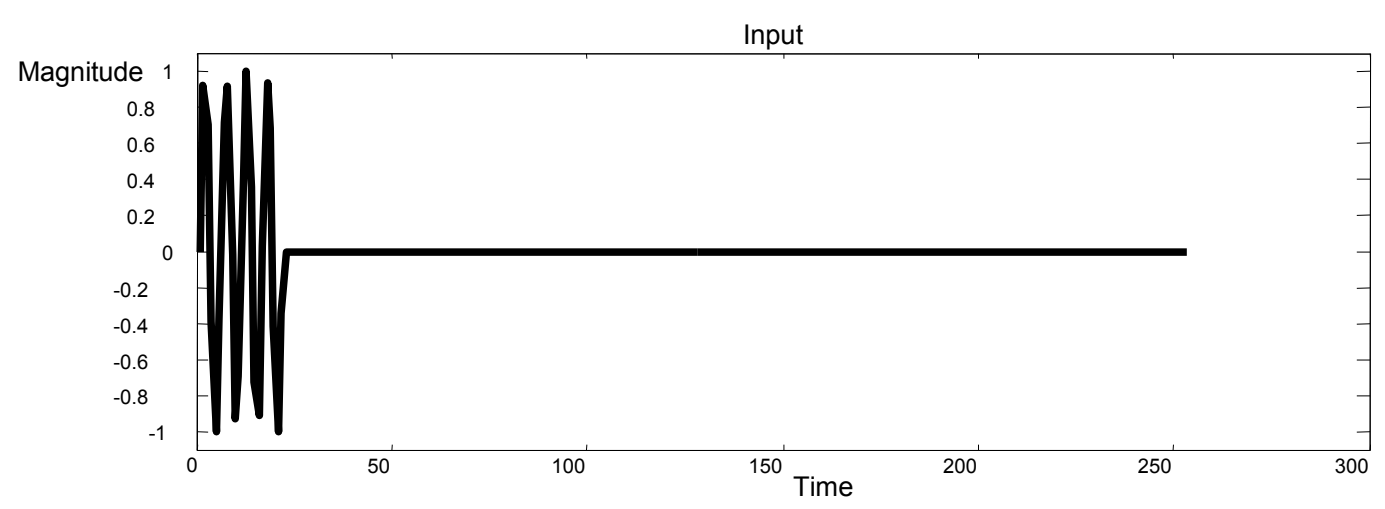

(a) Input of frequency $\mathrm{f}_{0}=5.4 \mathrm{GHz}$ and a pulse width $\delta=0.685 \mathrm{nsec}$.

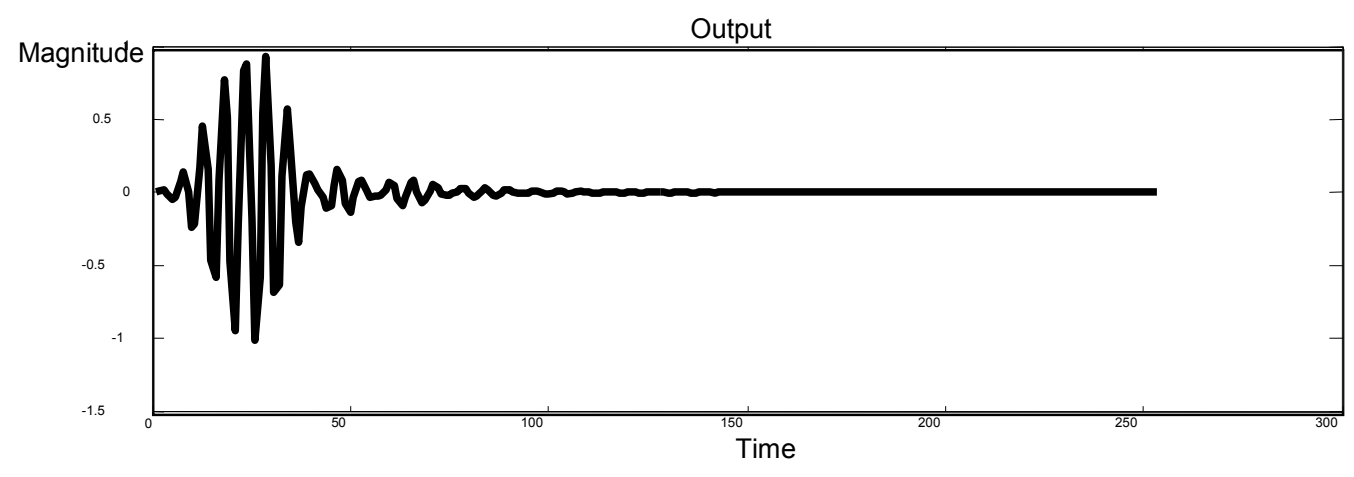

(b) Output of WDF.

Figure 6. Output of bandpass filter matched to a rectangular pulse 
It is clear from Figure 6 that the maximum output generated by this filter occurs at the delay time $\delta$ which indicates the completion of the arrival of input signal.

\section{Conclusion:}

We have presented a design procedure for the construction of single pulse matched filters for radar applications derived from arbitrary amplitude transfer functions.

The procedure has the following advantages:

- The design circuits can accurately approximate the $\sin (x) / x$ frequency response.

- The procedure is simple to apply.

We have also presented the Lattice Wave Digital Filters approach for design a bandpass filter specifications which cannot be obtained from low-pass filter frequency transformation. This approach is more applicable for any analogue circuit than that one which depends on the Ladder networks only.

\section{References:}

[1] Skolnk, M. I., Radar Handbook, Mc Graw-Hill, Inc., 1970.

[2] R.Nouta, and H.J.Linkklaen Arriëns, "On the use Use of the Sharpe Chebyshev Rational Function Approximation", Proc. Of ProRISC, 2002

[3] Fettweis, A.," Wave digital filters: theory and practice", Proceedings IEEE, Vol. 74, 270- 327, Feb. 1986.

[4] H.J. Lincklaen Arriëns, "Calculation of the Coëfficiënts of a Lattice Wave Digital Filter", Oct. 2003.

[5] Gazsi, L.,"Explicit formulas for lattice wave digital filters" , IEEE trans. Circuit \& Sys., Vol.32, 68-88, 1985.

[6] Lfred Fettweis and Klaus Meerkötter, "On adaptors for wave digital filters", IEEE transactions on acoustic, speech, and signal processing, Vol.ASSP-23, No.6, December 1975.

[7] K.H. Moustafa and M.I.Sobhy, "Lumped/Distributed Single Pulse Matched Filter for Radar Applications based on Wave Digital Filters", IEEE International Symposium on Circuits and Systems, PP.311-315, May.1999 